\title{
GOBERNAR A LA JUVENTUD VULNERABLE: UN ANÁLISIS DISCURSIVO DE LAS POLÍTICAS DE EMPLEO JUVENIL EN CHILE*
}

\author{
GOVERNING VULNERABLE YOUTH: AN ANALYSIS \\ OF THE YOUTH EMPLOYMENT POLICY DISCOURSE IN CHILE \\ Guillermo Rivera-Aguilera \\ Pontificia Universidad Católica de Valparaíso \\ griverov@gmail.com
}

\section{RESUMEN}

El presente artículo se pregunta cómo se gobierna la juventud vulnerable desde los discursos de las políticas de empleo juvenil en Chile. Para esto se realiza un análisis de discurso que toma como referencia documentos públicos elaborados entre los años de 2008 a 2015. Los resultados dan cuenta de cuatro hilos discursivos que emergen de las políticas de empleo juvenil: a) el discurso de la equidad-inequidad; b) el discurso del capital humano; c) la figura del joven vulnerable; d) la figura del joven productivo. De acuerdo con el análisis basado en el enfoque de la gubernamentalidad se discute en torno a elementos de la política que prescriben formas de "conducir la conducta» de los jóvenes vulnerables para su inclusión al mercado del trabajo.

PALABRAS CLAVE: gubernamentalidad, juventud vulnerable, políticas de empleo, análisis del discurso.

\section{ABSTRACT}

This article is an inquiry into how vulnerable youth have been governed from the perspective of the youth employment policy discourse in Chile. The analysis of this discourse was drawn from public documents drafted between 2008 and 2015. Four streams of ideas were identified in the Chilean youth employment policy: a) the equity-inequity discourse; b) the human capital discourse; c) vulnerable youth; and d) productive youth. From the standpoint of an analysis based on a governmentality approach, this article discusses the policy elements that prescribe forms of «conduct» for vulnerable youth and their inclusion into the labor market.

KEY WORDS: governmentality, vulnerable youth, employment policies, discourse analysis.

\footnotetext{
* El texto fue elaborado en el marco de mi pasantía de investigación doctoral en la School of Management de la Universidad
} de Leicester. Agradezco los aportes del doctor Dimitris Papadoupoulos, quien comentó versiones preliminares de este texto. 


\section{INTRODUCCIÓN}

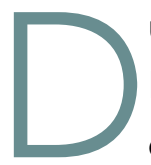

urante 2013, de acuerdo con datos oficiales de la Organización Internacional del Trabajo (OIT), 73 millones de jóvenes entre 15 y 24 años estaban desempleados (OIT 2013a). Desde estas cifras se pueden constatar una serie de vulnerabilidades en la población juvenil en lo tocante al empleo, principalmente porque los jóvenes corresponden al grupo etáreo más expuesto a quedar sin trabajo o a no acceder a este. En consecuencia, diversos organismos internacionales han insistido en la importante función de las políticas destinadas a aumentar tanto la cantidad como la calidad de los empleos para los jóvenes (OIT 2010, 2013a).

En el contexto regional, el informe «Trabajo decente y juventud en América Latina» (OIT 2013b) afirma que los jóvenes de esta parte del planeta enfrentan una tasa de desempleo casi tres veces más alta que la de los adultos y a la vez representan 40\% del desempleo. De los 108 millones de jóvenes en América Latina y el Caribe (entre 15 y 24 años de edad), 35\% estudian y $33 \%$ trabajan; cerca de $12 \%$ estudian y trabajan al mismo tiempo, y $20 \%$ ni estudian ni trabajan (principalmente mujeres jóvenes). Entre los que trabajan, un elevado porcentaje lo hace en condiciones precarias, es decir, más de la mitad (55.6\%) tienen un empleo informal, bajos ingresos, inestabilidad laboral y desprotección de derechos en ese contexto.

El escenario en Chile no es muy distinto de lo que ocurre a nivel de América Latina y el Caribe. Autores como Aguilera (2009), Velasco y Hunneus (2011) sostienen que en los últimos 30 años no se presentan resultados en las políticas juveniles orientadas al trabajo, ni a la inclusión social y económica de este grupo de la población, especialmente para aquellos sectores con mayor pobreza y vulnerabilidad. Parte importante de los pobres en Chile son mujeres o jóvenes, hecho que contribuye a acrecentar la desigualdad (Velasco y Hunneus 2011).

En este contexto, el segundo gobierno de la presidenta Bachelet ha puesto en marcha un nuevo programa de inserción laboral dirigido a mujeres y jóvenes en situación de vulnerabilidad social. Esta iniciativa se desarrolla como una de las medidas planteadas para sus primeros 100 días de gobierno. El programa denominado «Más Capaz» se ha planteado como objetivo aumentar la participación e inclusión de mujeres y jóvenes al mercado del trabajo desde un modelo de formación orientado a mejorar las competencias laborales (SENCE 2014).

En palabras de la propia presidenta Bachelet: 
Un gran motor de inclusión y equidad es incorporar al trabajo a quienes tienen mayores dificultades para hacerlo, estamos hablando de las mujeres y los jóvenes. En Chile, 49 de cada 100 jóvenes de entre 19 y 29 años no estudian ni trabajan. Es una de las cifras más altas de la región. Son más de 770 mil jóvenes. Por otro lado, sólo el 48 por ciento de las mujeres chilenas tienen un trabajo remunerado. Entre los compromisos de gobierno que tomamos para los primeros 100 días, están las medidas que apoyan el acceso y permanencia en el mercado laboral de mujeres y jóvenes. Serán más de 450 mil beneficiarios los que podrán acceder a una beca para capacitarse en un oficio que les permita acceder a un trabajo. Al año 2018 esperamos haber llegado a 450 mil beneficiarios, estamos hablando de 300 mil mujeres entre 30 y 60 años, dentro de las cuales se consideran 40 mil mujeres para trabajos de microemprendimiento y a 150 mil jóvenes entre 19 y 29 años. [...] Nuestro compromiso es que, al final de este gobierno, gracias a este programa de capacitación ambicioso, más mujeres, jóvenes y personas jóvenes con algún tipo de discapacidad, sean dueños de mejores herramientas para acceder a un trabajo de calidad (Mensaje Presidencial, Gobierno de Chile 2014:26).

De acuerdo con la literatura revisada, programas como Más Capaz corresponden a un ejemplo de las estrategias desarrolladas a nivel global para hacer frente al problema del empleo-desempleo, en un contexto de poscrisis económica. De hecho, se puede constatar cómo la ejecución de programas locales de empleo orientados a la población juvenil son similares en países tan distintos como Argentina, Suecia, Reino Unido (Assusa y Brandán 2014, Chertkovskaya et al. 2013, Holmqvist et al. 2012). Estas políticas revelan la generación de dispositivos homogéneos para contrarrestar la problemática del desempleo y la inactividad de jóvenes que no trabajan ni estudian, por medio de un discurso que promueve una «ciudadanía activa» (Dean1995, Kotkas 2010).

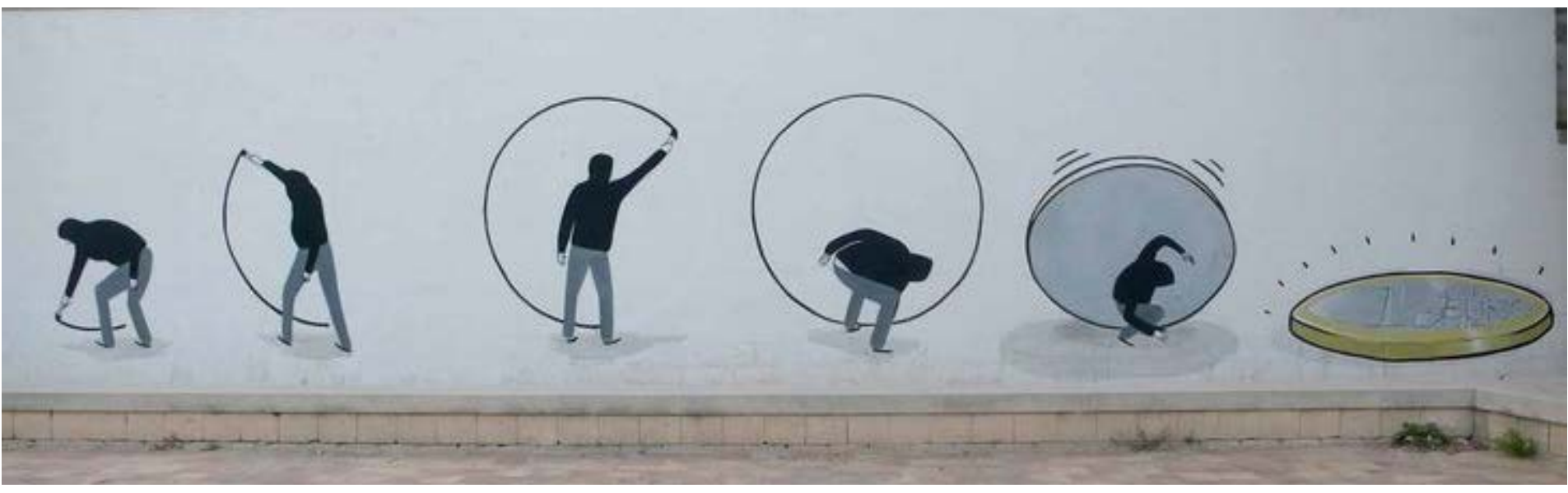


Estas formas de entender las políticas de empleo juvenil se sitúan en un contexto internacional y se apoyan en lineamientos de instituciones como el Banco Mundial y la Organización Internacional del Trabajo (Rivera-Aguilera 2015). En esta línea, algunos autores (Kaasch 2013) hablan de «políticas sociales globales» que promueven una serie de conceptualizaciones, normas, orientaciones y métodos para ser aplicados a niveles locales.

En esta investigación nos interesa centrarnos en cómo las políticas de empleo en Chile generan un discurso basado en la empleabilidad (Rentería y Malvezzi 2008) desde la promoción de una ciudadanía activa y productiva. Vemos que por medio de programas como Más Capaz se interviene y administra la problemática del empleo-desempleo, orientados a una población especifica de jóvenes: los más vulnerables.

Analizaremos, entonces, las políticas de empelo en Chile desde una perspectiva que reflexiona sobre las formas de gobierno de la juventud. La pregunta estará centrada en el «cómo se gobierna» (Dean 1995), considerando que en las últimas décadas se ha desarrollado un campo de creciente fuerza en las ciencias sociales que se ha denominado «Estudios de la gubernamentalidad», el cual sobresale por su insistencia en entender cómo somos gobernados y en la relación existente entre gobierno y conducta. Este artículo tomará como referencia esa perspectiva para reflexionar en torno a los discursos existentes en las políticas y los programas de empleo juvenil. Esto desde la inquietud por entender cómo se gobierna a la juventud vulnerable desde los discursos que emergen de las políticas de empleo en Chile.

\section{ESTUDIOS DE LA GUBERNAMENTALIDAD Y EL TRABAJO}

Una serie de publicaciones han descrito que los «estudios de la gubernamentalidad» tienen sus orígenes en las ideas sobre el poder y las formas de gobierno de las poblaciones desarrolladas por Michel Foucault $(2009,2012 a)$ en los cursos «Seguridad,territorio y población» (2009[1978]) y«El nacimiento de la biopolítica» (2012a[1979]), impartidos en el Collège de France (Burchell et al.1991, CastroGómez 2010, Flew 2014, Grinberg 2007, Miller y Rose 2009, Rose, O’Malley y Valverde 2012, Senellart 2009, Vargas-Monroy y Pujal 2013).

Desde los estudios de la gubernamentalidad se desprende un desarrollo en la literatura de las ciencias sociales y en la teoría política. Siguiendo a Mitchell 


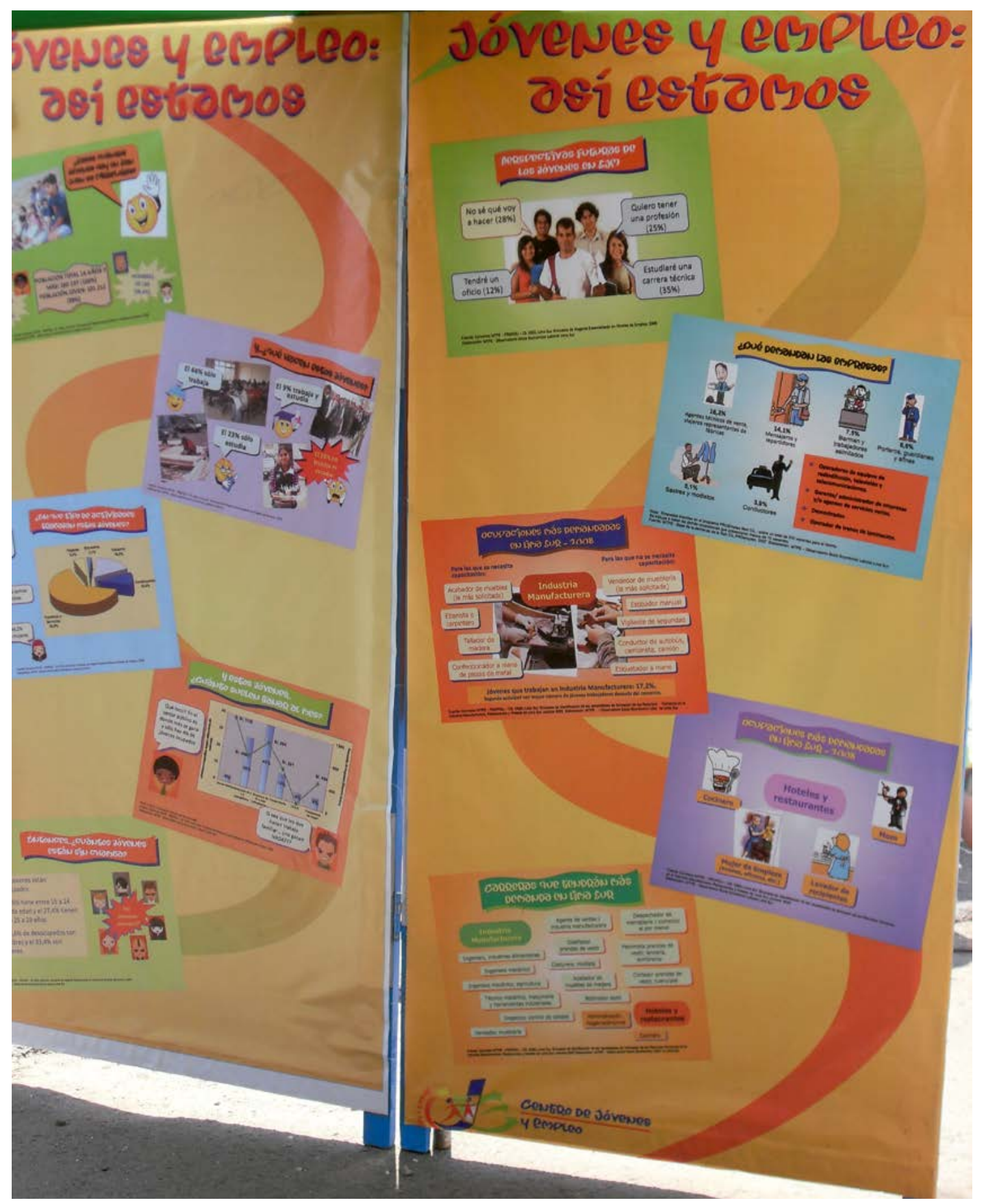

Dean (2007), el trabajo producido en las últimas décadas puede ser visto como si formara una nueva subdisciplina de las ciencias sociales y las humanidades, preocupada por el «cómo» del gobierno. En esta línea, Rose et al. (2012) afirman que el enfoque sobresale por «su insistencia de entender cómo somos gobernados en el presente individual y colectivamente, en nuestros hogares, escuelas, 
hospitales, ciudades, regiones y naciones, tanto por parte de nuestros cuerpos de gobierno nacionales así también por parte de los transnacionales» (Rose et al. 2012:143).

La pregunta está centrada en el cómo y no en el qué se gobierna. Tal como sostiene Dean (1995), los estudios se centran en el cómo se conduce la conducta de los sujetos como forma de gobierno de las poblaciones. Estas formas de entendimiento han permitido «iluminar aspectos de nuestra vida social y política, particularmente aquella que concierne al sentido de conducta de la conducta, particularmente, la relación entre gobierno y conducta» (Dean 1995:560).

Si bien los estudios de gubernamentalidad fueron inicialmente cuestionados por su poca aplicación empírica y por tener características eurocéntricas, en los últimos años se han desarrollado una serie de investigaciones sobre el trabajo y el empleo desde este enfoque (Rose 1996, Grinberg 2007, Vargas-Monroy y Pujal 2013, Mussetta 2009). Tales estudios empíricos se desarrollan principalmente desde el mundo anglosajón (Chertkovskaya et al. 2013, Miller y Rose 2009), escandinavo (Diedrich y Styhre 2013, Fejes 2010, Garsten y Jacobsson 2013, Vesterber 2013, Holmqvist et al. 2012) y existe una corriente emergente desde Latinoamérica (Assusa y Brandán 2014, Brandán2014, Castro-Gómez 2010, Pulido-Martínez 2012, 2008, Sáenz y Granada 2013, Vargas-Monroy y Pujal 2013).

El hecho de analizar cómo se construye una forma de gobierno respecto de la población juvenil a partir de los discursos que elaboran las políticas de empleo en Chile, es la propuesta que explora este artículo. Siguiendo las ideas de Foucault sobre la gubernamentalidad, las políticas de empleo juvenil se desarrollan desde una racionalidad centrada en «conducir la conducta» de los jóvenes. Esto con la finalidad de hacer que ejerzan su libertad de modos específicos, basados en los objetivos estratégicos de programas de empleo y del mercado del trabajo (Castro-Gómez 2010, Du Gay 1996, Stecher y Godoy 2014).

\section{NEOLIBERALISMO Y TECNOLOGÍAS DE GOBIERNO}

Siguiendo con las ideas de Foucault, desarrolladas en el curso «El nacimiento de la biopolítica», de 1979, entenderemos el neoliberalismo como un «arte de gobernar». Sus orígenes obedecen a una racionalidad proveniente del liberalismo (Foucault, 2012a[1979], Flew 2014, Castro-Gómez 2010, Miller y Rose2009). 
Para este estudio entenderemos el neoliberalismo como «una ideología y una práctica política. Sostiene que el bienestar y los bienes sociales serían maximizados si el mercado se extiende desde la noción de propiedad a cada esfera de la interacción humana» (Tadajewsky et al. 2011:173).

Esta ideología y práctica política de orden neoliberal se materializa en una serie de discursos que prescriben las formas de entender los modos de producción y del trabajo en nuestras sociedades actuales. Es más, tal como señalan Rose (1996), Du Gay (1996), Parker et al. (2014), la ideología repercute también en las subjetividades, ya que construye un tipo de ser humano: un sujeto libre, un homo economicus, un trabajador flexible. Estas figuras de subjetividad son centrales en la ideología neoliberal y en las formas de gobernar a la población juvenil desde lineamientos globales (Parker et. al. 2014, Rivera-Aguilera 2015).

En esta línea, entenderemos la prescripción de las políticas orientadas a una ciudadanía activa y a un joven productivo como una conceptualización normativa basada en una racionalidad neoliberal, que se materializa en «figuras de la subjetividad» (Rivera-Aguilera 2015). Las figuras de la subjetividad corresponden a tecnologías de gobierno (Castro-Gómez 2010, Foucault 2012b) que prescriben formas de «conducir la conducta» de la juventud para su inclusión en los mercados del trabajo. Estas se estructuran en formas binarias e incluyen elementos normativos, que permiten la construcción de un adulto productivo. Como se ha constatado en un estudio anterior en relación con las figuras de la subjetividad que emergen de las políticas globales de la $\mathrm{OIT}^{2}$ (Rivera-Aguilera 2015), estas formas de clasificar a la población juvenil involucran una serie de elementos de valoración basados en principios economicistas y neoliberales. Parten de una serie de variaciones discursivas que se van desarrollando conforme a los discursos de las políticas de empleo juvenil que van cambiando de acuerdo con los ritmos del mercado del trabajo.

En relación con los conceptos y las perspectivas teóricas planteados, esta investigación entiende las políticas públicas orientadas al empleo juvenil como el resultado de un accionar de una serie de instituciones que interpelan al joven

\footnotetext{
${ }^{1}$ Autores como Flew (2014) han señalado que es mucho lo que se ha escrito sobre neoliberalismo en los últimos años y advierten que las definiciones de este concepto varían enormemente desde distintas disciplinas y perspectivas. En sus propias palabras el neoliberalismo es un «concepto muy invocado pero muy mal definido» (Flew 2014:53).

2 En el estudio señalado se mencionan seis figuras de la subjetividad que construyen al joven trabajador: a) el joven pobre-el joven no pobre, b) el joven ocioso-el joven productivo, c) la generación perdida-la generación no perdida, d) el joven feliz-el joven infeliz, e) los desafortunados jóvenes-los afortunados jóvenes y f) los jóvenes desconfiados-los jóvenes confiados.
} 
como sujeto productivo y en determinado momento prescriben formas de diagnosticar y normalizar a la juventud. Tal como ha señalado Fejes (2010), estos criterios pueden ser analizados desde documentos oficiales de uso público. Para esto se desarrolla un análisis documental (Atkinson y Coffey 2006) que toma como referencia informes, leyes y documentos programáticos asociados con las políticas de empleo y juventud en Chile, desde el año 2008 a la fecha. Lo que se presenta a continuación es el resultado del análisis documental mencionado.

\section{MÉTODO}

Para ilustrar las formas en que las políticas de empleo justifican en forma discursiva los elementos normativos orientados ala juventud vulnerable en Chile, se realizó un análisis crítico del discurso (Parker 1996, Fairclough 2010, Fairclough y Wodak 2000, Jäger 2003, Stecher 2010, 2014). Se utilizó como técnica el análisis documental (Atkinson y Coffey 2006), desde el cual se entienden los distintos informes de la política como actores que legitiman autoridad y validan formas de poder, permitiendo estandarizar y categorizar a la juventud en relación con el empleo.

Siguiendo a Atkinson y Coffey (2006), asumiremos los documentos como actores, es decir, como objetos que desarrollan cosas. Legitiman la autoridad y validan formas de poder. En este sentido, la relevancia del análisis radica en que los textos, sean documentos oficiales o discursos de autoridades, sirven para presentar una lógica de gestión, actuar en el terreno local, interpelar a los actores, moldear relaciones, prescribir acciones, permitiendo de esta manera estandarizar y categorizar a los sujetos. Así, para esta investigación la clave del papel de los documentos es que estandarizan y categorizan a la población juvenil.

Los discursos sobre juventud pueden dilucidarse desde documentos que realiza la política pública sobre empleabilidad juvenil. Algunos ejemplos de este tipo de discurso son los debates parlamentarios, los documentos de prensa, informes oficiales en materia de procesos gubernamentales y discursos de expertos, que se basan en investigaciones y datos empíricos relacionados con la problemática (Sisto y Fardella 2011). En esta línea, nos interesa profundizar en la justificación de la política, es decir, qué discursos justifican y difunden los diseños de políticas.

Para este estudio se seleccionaron como corpus nueve documentos públicos sobre políticas de empleo juvenil en Chile (cuadro 1). Estos incluyen informes de 
comisiones de expertos, proyectos de ley y mensajes presidenciales desarrollados entre los años 2008 y 2015.

Cuadro1. Documentos seleccionados para este estudio

- Consejo Asesor Presidencial (2008): Informe Trabajo y Equidad: Hacia un Chile más justo: Trabajo, salario, competitividad y equidad social.

- Congreso Nacional (2009a): Mensaje de S.E la Presidenta de la República con que inicia un proyecto de ley que crea el subsidio al empleo.

- Congreso Nacional (2009b): Cámara de Diputados. Discusión en sala Subsidio al empleo juvenil. Primer trámite constitucional. Legislatura 357, Sesión 02. 12 de marzo de 2009.

- Congreso Nacional (2009c): Oficio de Ley a S.E. la Presidenta de la República, comunica texto aprobado por el Congreso Nacional. 19 de marzo de 2009.

- Ministerio del Trabajo y previsión Social (2009): Ley $N^{\circ} 20.338$ Crea el subsidio al empleo. Publicación Diario Oficial. 1 de abril de 2009.

- Congreso Nacional (2009d): Firma proyecto de Ley Subsidio al empleo. Intervención de S.E la Presidenta de la República Michelle Bachelet, en acto de firma del proyecto de ley sobre subsidio al empleo.

- $\quad$ Gobierno de Chile (2014): Mensaje Presidencial. 21 de mayo de 2014.

- $\quad$ Servicio Nacional de Capacitación y empleo (2015). Guías operativas 2015. Programa Más Capaz.

- $\quad$ Gobierno de Chile (2015): Mensaje Presidencial. 21 de mayo de 2015.

De acuerdo con Sisto y Fardella (2011), el tipo de documentos públicos aquí seleccionados resulta fuente de gran riqueza para mostrar el funcionamiento de la retórica justificativa con la cual se instalan transformaciones en política pública, ya que interpelan las identidades de los actores sociales involucrados.

Del corpus analizado se seleccionaron inicialmente 19 citas que daban cuenta de cómo se justifican discursivamente los elementos normativos existentes en las políticas de empleo. Estas se ordenan y presentan desde dos ejes. El primero «descriptivo-secuencial», denominado «Equidad social: la juventud vulnerable como capital humano» da cuenta de cómo se van desarrollando una serie de transformaciones en las políticas del trabajo en Chile, que tienen como repercusión una serie de variaciones discursivas en torno a las conceptualizaciones de la población juvenil, desde el año 2008 a la fecha. Para ejemplificar este proceso se presentan tres citas, ordenadas cronológicamente. 
El segundo eje "analítico» se ha denominado «La figura del joven vulnerable y la figura del joven productivo». Este eje se centra en los hilos discursivos (Fairclough 2010) que emergen en torno a la equidad social y la figura del joven productivo como tecnología de gobierno.

Finalmente, se plantea una discusión de cómo los resultados presentados contribuyen a responder a la pregunta de investigación.

\section{RESULTADOS \\ EJE1.EQUIDAD SOCIAL: LA JUVENTUD VULNERABLE COMO CAPITAL HUMANO}

Durante el primer gobierno de Bachelet (2006-2010) se crea y se pone en operación el «Consejo Asesor Presidencial Trabajo y Equidad» (CAPTE). Este estamento público-privado es conformado por distintos actores pertenecientes al Estado, a la sociedad civil y al sector empresarial. En el informe desarrollado por esa comisión se plantea la necesidad de diseñar una nueva y «mejor política» para el fututo y el progreso del país. La denominada «política social y laboral para un nuevo siglo» se basa en un discurso centrado en el crecimiento económico, la reconversión laboral y una reactualización permanente de toda fuerza de trabajo (CAPTE 2008).

La cita número 1 es tomada del informe del CAPTE (2008) e ilustra la importancia de la equidad en las nuevas políticas sociales y del trabajo.

\section{Cita 1}

Una sociedad con inequidades sin resolver es un campo propicio para la aparición de discursos confrontacionales, climas de desconfianza y enfrentamiento. Estas situaciones perturban nuestra convivencia, perjudican la consolidación de un sistema armónico de relaciones laborales e incluso, en el mediano plazo, pueden afectar las condiciones de competitividad de nuestra economía y su potencial crecimiento.

El desarrollo del capital humano, mediante la educación, la capacitación, la certificación del conocimiento, es en el largo plazo determinante para incrementar la productividad y los salarios en la economía chilena. Al mismo tiempo, el gobierno está llevando a cabo un enorme esfuerzo para cumplir su compromiso de construir un sistema de protección social que dé seguridad a las personas. Ello se manifiesta en las reformas de previsional, de infancia y salud, y también debe expresarse en otras políticas di- 
rigidas a mejorar la distribución de ingreso y favorecer un mercado del trabajo más moderno y equitativo.

Lograr una mayor equidad en el mundo del trabajo es parte de esta tarea. Es un sentido anhelo de la ciudadanía que se adopten medidas para fortalecer los ingresos de las familias, fomentar una mayor capacitación y productividad de las personas, garantizar el trabajo digno y promover relaciones laborales equilibradas y constructivas (CAPTE 2008:11).

La cita 1 refleja una nueva forma de entender las políticas del trabajo en Chile. Vemos que la equidad se plantea como necesaria para un orden social. En términos productivos permite un «sistema armónico de relaciones laborales» y, por lo tanto, una mayor competitividad que contribuya al crecimiento económico. En esta línea, la amenaza de la inequidad puede favorecer «discursos confrontacionales» que atenten con el desarrollo óptimo del país.

En el segundo párrafo se plantea el desarrollo del capital humano (Becker 1993) desde la capacitación y la «certificación del conocimiento» como el camino o la vía que permitirá, en el largo plazo, «incrementar la productividad y los salarios de la economía chilena». Para esto es necesario educar a la población desde lineamientos específicos, basados en conocimientos estandarizados y certificados, los cuales aumentarán la productividad y por ende los salarios de la economía chilena. Podemos entender de esto último que no están garantizados ni los salarios ni el empleo ya que, de acuerdo con el fragmento, los salarios aumentarán solo si la economía crece. En consecuencia, el mandato es que todos los actores del mercado del trabajo tienen que ser activos en esta tarea. Es decir, es tarea de todos los ciudadanos que Chile sea un país productivo, de lo contrario no habrá aumento en los salarios y en consecuencia no habrá equidad social. El hecho de no tener un país equitativo puede perturbar nuestra convivencia y paz social.

El fragmento seleccionado también plantea como elemento fundamental un sistema de protección social que permita seguridades individuales en materias de previsión social y salud. En el ámbito del trabajo corresponden a «políticas dirigidas a mejorar la distribución del ingreso y favorecer un mercado del trabajo más moderno y equitativo». Desde esta afirmación podemos dar cuenta cómo el discurso de la equidad aparece nuevamente, ahora basado en la importancia de la distribución del ingreso. Esto corresponde a un discurso que apela a una sociedad funcional, en paz, que vaya en armonía con los lineamientos de una economía más global. 
Desde lo anterior podemos entender que la equidad social planteada por la política corresponde a una normativa donde el trabajo «digno» solo será posible si la ciudadanía es parte de programas como Más Capaz, ya que ofrecen «una mayor capacitación y productividad de las personas».

En la siguiente cita vemos cómo estas trasformaciones del trabajo repercuten directamente en las políticas de empleo juvenil. Para esto hemos seleccionado un fragmento de la Ley Subsidio al empleo juvenil, ${ }^{3}$ la cual se centra en la capacitación de los jóvenes más vulnerables del país. A partir de esta disposición se plantea la necesidad de formalizar el trabajo de los jóvenes chilenos con el fin de aumentar el capital humano para un mayor crecimiento económico. De esta manera se promueve que la «juventud vulnerable» se inserte en el mercado del trabajo y así contribuyan al crecimiento económico del país como sujetos productivos.

\section{Cita 2}

«La necesidad de focalizar el subsidio en los jóvenes más vulnerables»

Existen más de 768000 chilenas y chilenos que son jóvenes entre 18 y 24 años que pertenecen a los sectores más vulnerables de nuestro país. Ellos conforman un sector de nuestra sociedad que enfrenta enormes dificultades ante el desafío de trabajar. La tasa de participación laboral de los jóvenes del quintil más pobre del país es un 60 por ciento inferior a la del quintil más rico. Los jóvenes carecen de experiencia laboral y muchos de ellos también carecen de habilidades certificadas, lo que perjudica sus potencialidades de productividad. Esta realidad es especialmente preocupante en los hogares más vulnerables, donde las habilidades productivas de los diferentes oficios aún son transmitidas a los jóvenes por sus padres o por sus familiares directos o tutores, que a su vez también han tenido trabajos informales, de forma tal que se reproduce el círculo de la informalidad y la precariedad social. Además, los jóvenes más vulnerables tienen serias dificultades para encontrar trabajo, ya que carecen de redes laborales. Por otra parte, un porcentaje importante de los jóvenes carecen de los hábitos y de la disciplina necesaria para mantener y progresar en un trabajo subordinado, a lo cual se suma la falta de apoyo de la sociedad para ayudar a definir el tipo de trabajo que se acomoda más a sus expectativas y preferencias. Por último, es inevitable consignar que los primeros salarios que reciben los jóvenes que pertenecen a los hogares más vulnerables son sustancialmente menores que los que reciben los jóvenes de familias

3 La Ley 20338 se tramitó en tiempo récord. Desde un discurso de unidad nacional, en un contexto de crisis económica mundial se destinan 100 millones de dólares para distribuir entre la capacitación de los jóvenes y las empresas que los contrataran. 
de mayores ingresos, lo que en muchos casos desincentiva la búsqueda de un trabajo formal o la permanencia en el mismo. Estas dificultades generan los incentivos para la búsqueda de otras fuentes de ingresos, tanto en el sector informal o en otras actividades que pueden llevar al joven incluso a la delincuencia, con las graves consecuencias personales, familiares y sociales que esto acarrea. Estos factores inciden en que este grupo de la población se demore más tiempo en encontrar un empleo, e incide en que muchos jóvenes se vean tempranamente desalentados en sus intentos de encontrar empleo. Todas estas dificultades de acceso han provocado una persistente baja en la participación laboral de los jóvenes, con la consecuente exclusión productiva y social, además de una alta rotación laboral (Mensaje de la Presidenta para iniciar proyecto de ley que crea el subsidio al empleo. Congreso Nacional 2009a:5).

Esta cita permite ilustrar que desde un discurso centrado en la carencia, la informalidad, la inequidad y la delincuencia se va caracterizando un tipo de joven que se hace necesario focalizar como una población específica en relación con el trabajo: «los jóvenes más vulnerables». Estos se caracterizan por ser «jóvenes carentes», ya que no tienen experiencia laboral, no poseen habilidades sociales para el empleo ni redes sociales necesarias para insertarse en el mercado laboral.

Este joven vulnerable se asocia con un trabajador informal. Sus «habilidades productivas» son transmitidas desde la familia de origen. Desde este argumento podemos entender que la familia obedece al actor responsable de la informalidad del trabajo de estos jóvenes, hecho que explica la reproducción de un «círculo de la informalidad y precariedad social», que se hace necesario erradicar desde los lineamientos de las nuevas políticas de empleo.

Por otro lado, vemos cómo emerge un discurso centrado en la normalización y basado en el argumento de que «los jóvenes carecen de los hábitos y de la disciplina necesaria para mantener y progresar en un trabajo subordinado». Este tipo de afirmaciones se hacen relevantes para comprender la forma en que se está prescribiendo la inclusión laboral del joven trabajador chileno. A partir de lo anterior podemos dar cuenta cómo el discurso empresarial se hace presente en el diagnóstico de la política, emitiendo una normativa hacia el joven que busca empleo desde dos características explicitas. En primer lugar, debe tener «la disciplina necesaria», asociada con una serie de habilidades blandas, que de acuerdo con lbáñez (2005) no son transmitidas en la educación formal ni tampoco por las familias de origen. En segundo lugar, el joven vulnerable como usuario del subsidio está caracterizado para transformarse en un trabajador subordinado. 
En el fragmento 2 emerge un discurso centrado en la inequidad que plantea algunas contradicciones de la realidad chilena. Principalmente desde la problemática del joven vulnerable que se trasforma en trabajador que obtiene una retribución económica muy baja en comparación con otros jóvenes chilenos de clases medias y altas. Estas consecuencias de la inequidad que enfrenta el joven vulnerable lo identificarán como un joven trabajador pobre, teniendo como efecto la posible deserción del mercado del trabajo y por lo tanto dejar de ser un joven productivo. Ahora bien, en esta oscilación entre inequidad y la posible amenaza de que el joven deje de ser productivo, emerge el discurso de la delincuencia. Esta asociación de la juventud vulnerable al margen de la legalidad la podemos ejemplificar desde afirmaciones como: «Estas dificultades generan los incentivos para la búsqueda de otras fuentes de ingresos, tanto en el sector informal como en otras actividades que pueden llevar al joven incluso a la delincuencia, con las graves consecuencias personales, familiares y sociales que esto acarrea». En función de lo expuesto podemos entender que el joven vulnerable se encuentra en un límite, es decir, se encuentra entre una inserción laboral «precaria» (Standing 2009) o en el riesgo de convertirse en un joven no productivo para la sociedad.

A continuación veremos cómo estas políticas se materializan en un nivel programático. La siguiente cita es parte de un manual del programa Más Capaz que está dirigido a los profesionales y técnicos que trabaja con los jóvenes más vulnerables. Como podemos ver, el fragmento corresponde a una definición de juventud que incluye elementos diagnósticos para un trabajo de intervención social con la población juvenil.

Cita 3

Los(as) jóvenes

Muchos jóvenes carecen hoy de las habilidades fundamentales requeridas en el mundo laboral actual, que los conviertan en candidatos atractivos a diversos puestos de trabajo. Estos no cuentan con las habilidades técnicas, ni otras habilidades caracterizadas como «habilidades blandas, transversales o para la vida», como por ejemplo: la comunicación asertiva, la confiabilidad y la capacidad para trabajar en equipo, por mencionar algunas. Todas ellas, habilidades que articuladas a los conocimientos, son necesarias en cualquier tipo de trabajo (Guía Operativa Programa Más Capaz, SENCE 2015:5). 
En esta cita podemos dar cuenta de cómo se concibe al joven como extremadamente carente de recursos para enfrentar el mundo productivo. Desde esta conceptualización se les considera con una total ausencia de «habilidades fundamentales requeridas en el mundo laboral actual», hecho que no los hace «candidatos atractivos» para acceder a puestos de trabajo. Es más, el joven vulnerable es un sujeto que no tiene «habilidades para la vida». A partir de lo anterior podemos entender que el joven usuario de los programas de empleo corresponde a un joven totalmente analfabeto del discurso empresarial. Por lo tanto, el joven vulnerable debe incorporar el discurso del mercado como un nuevo idioma, y este nuevo lenguaje es su puerta de entrada para su inclusión social, ya que es la única forma posible que tendrá para buscar empleo y poder ser contratado por algún empleador.

Desde la definición de «Los(as) jóvenes» del programa Más Capaz se puede ilustrar cómo los programas de empleo se encargan de alfabetizar en el discurso empresarial al joven vulnerable, ya que este tiene el «deber» de desarrollar una serie de habilidades que le permitan no solo hablarlo, sino también actuarlo o en términos performativos. Ello se logrará, incorporando el desarrollo de habilidades como la «comunicación asertiva», la «confiabilidad» y desarrollar la «capacidad para trabajar en equipo». La adquisición de este nuevo lenguaje (verbal y performativo) corresponde a un elemento normativo que le permitirá al joven el disciplinamiento necesario para su inserción laboral.

A continuación presentaremos un análisis que incluye elementos transversales de las citas presentadas.

\section{EJE 2. LA FIGURA DEL JOVEN VULNERABLE Y LA FIGURA DEL JOVEN PRODUCTIVO}

En los fragmentos revisados en el apartado anterior hemos podido ilustrar que en los discursos de empleabilidad juvenil en Chile emergen una serie elementos normativos de manera transversal. A continuación desarrollaremos un análisis que se detiene en identificar hilos discursivos (Fairclough 2010) que están presentes en el análisis de las políticas de empleo juvenil y que permiten «conducir la conducta» de los jóvenes vulnerables.

Un primer hilo discursivo en las políticas de empleo, corresponde a la dicotomía equidad-inequidad. De acuerdo con la cita 1 podemos entender que el 
significado de la equidad no opera como un valor por sí mismo, sino más bien como una «herramienta» que permite construir una retórica basada en el aumento de la productividad y el crecimiento económico. Ello queda de manifiesto si tomamos, por ejemplo afirmaciones tales como «una sociedad con inequidades sin resolver es un campo propicio para la aparición de discursos confrontacionales», que no permitirá un «sistema armónico de relaciones laborales» y limitará las posibilidades de ser un país competitivo que crece económicamente. De esta manera, podemos entender el discurso de la equidad como una «herramienta» que facilita reducir el conflicto en términos de un mayor equilibrio social, hecho que en términos del sociólogo Norbert Elias permite «una pacificación de la sociedad» (Deacon 2007).

Desde esta perspectiva, el discurso centrado en la equidad permite gobernar desde la paz social. En esta línea podemos entender la equidad como un elemento que favorecerá la producción y el crecimiento económico del país. Para esto se hace fundamental promover una «ciudadanía activa», que trabaje y sea productiva, dejando de esta forma atrás las políticas asistenciales.

Un segundo hilo discursivo corresponde a las formas de entender el empleo desde la perspectiva del capital humano. A través de estos lineamientos de la política centrada en la equidad, vemos la emergencia del discurso del capital humano como la retórica justificativa que promueve capacitar a la población juvenil. La prescripción que plantea la política es que el joven para acceder al trabajo formal está obligado a capacitarse, por ende, debe incorporar una serie de competencias basadas en habilidades técnicas y en blandas que le permitan desarrollar hábitos y «la disciplina necesaria para mantener y progresar en un trabajo subordinado» (cita 2).

En los fragmentos seleccionados hemos ilustrado cómo en los nuevos lineamientos de la política desarrollada a partir del CAPTE (2008) se plantea explícitamente el trabajo como una mercancía, donde la educación centrada en la productividad adquiere mayor relevancia, transformándose en la puerta de entrada para la inclusión social de los jóvenes chilenos de sectores bajos. Vemos que a partir de ahora, la capacitación y la certificación de conocimientos corresponden a elementos estandarizadores para que la población adquiera el lenguaje de los mercados y por lo tanto se pueda insertar laboralmente. El mandato de las políticas de empleo es que toda la población trabaje, lo cual facilitará nuestra convivencia como país y a su vez aumentará la competitividad de nuestra economía y por lo tanto el potencial de crecimiento económico del país. 
Una tercer hilo discursivo corresponde a las conceptualizaciones que se hacen de joven vulnerable, ya que de acuerdo con los documentos revisados, las formas de entender la vulnerabilidad desde las políticas de empleo significan al joven vulnerable como producto de su «ser». Es decir, desde su «ser» pobre, su «ser» excluido, su «ser» carente. Esto quiere decir que la vulnerabilidad para las políticas de empleo está puesta en «el sujeto». En esta línea y según las citas presentadas y los documentos analizados, las políticas no hacen un cuestionamiento o crítica respecto de cómo la inequidad produce vulnerabilidad. Tampoco se hace referencia a elementos estructurales que definen condiciones precarias de trabajo o la inactividad que se materializa, a través de jóvenes que no trabajan ni estudian.

Un cuarto hilo discursivo corresponde a la figura del «joven productivo». Para entender esta figura como una tecnología de gobierno, se hace necesario entender que la juventud económicamente activa es una ganancia económica para los países, mientras que la juventud que no trabaja es una pérdida de la inversión del Estado en materia educativa (Rivera-Aguilera 2015, OIT 2006). Vemos cómo la normativa actual plantea que el joven que no produce o que amenaza con no hacerlo es asociado a un joven que se encuentra en el límite de la delincuencia y caracterizado como un sujeto con potenciales conductas antisociales, "con las graves consecuencias personales, familiares y sociales» que ello conlleva.

Esta forma de entender al joven que no produce se caracteriza por asumir al sujeto que no trabaja como un ciudadano costoso, ya que no contribuye al bienestar social. En términos de inversión pública es necesario invertir en este sujeto, por medio de programas sociales relacionados con seguridad pública y salud mental.

Según este análisis, el «joven vulnerable» obedece a una población juvenil que debe ser guiada y conducida en una lógica de pastoreo (Foucault 2009) para salir de su condición actual e incorporarse en el mercado del trabajo desde un empleo formal. El joven vulnerable se encuentra entonces en un límite difuso, en el cual necesariamente debe incorporar este lenguaje empresarial, tanto en sus discursos como en sus conductas, ya que si no lo hace no podrá transformarse en sujeto productivo y, por lo tanto, se le cerrarán las puertas de la inclusión social. 


\section{REFLEXIONES FINALES}

El presente estudio se ha preguntado cómo se gobierna la población vulnerable desde las políticas de empleo juvenil en Chile. A partir de la revisión de documentos públicos se han podido constatar una serie de discursos que emergen desde distintos actores y que configuran una serie de hilos discursivos que permiten un entendimiento, desde el enfoque de la gubernamentalidad, respecto de las formas de gobierno de un segmento específico de la población juvenil.

Los resultados obtenidos dan cuenta de que las políticas de empleo juvenil se basan en un discurso centrado en la equidad social que promueve la inclusión social de jóvenes vulnerables, a través de su capacitación en programas que fomentan las competencias laborales desde una lógica del capital humano. Así, el joven vulnerable, para tener una posibilidad de acceder a la inclusión social a través del empleo formal, debe ser disciplinado y normalizado desde un discurso empresarial.

Hemos visto que las nuevas políticas promueven un joven productivo, capacitado y por lo tanto empleable para asumir un rol de subordinado. Esto significa que las políticas de empleo prescriben que el joven que se encuentra en una situación de vulnerabilidad tiene el deber de transformarse en un adulto productivo como parte de su rol social. Debe entonces reconocer e incorporar este mandato social y hacerlo parte de su subjetividad. Los ejes analíticos desarrollados permiten entender cómo se constituye un tipo de subjetividad basada en una serie de elementos normativos que se explican con las figuras de la subjetividad, específicamente con las dicotomías «joven vulnerable-joven productivo».

Finalmente, a lo largo de este artículo hemos podido dar cuenta de que partir del año 2008, con los lineamientos del Consejo Asesor Presidencial, emergen nuevos discursos de las políticas de empleo en las formas de conceptualizar al joven vulnerable. Hemos podido ilustrar, por medio de los estudios de la gubernamentalidad, una comprensión distinta al discurso oficial respecto de la juventud en Chile. Se sugiere que para futuros estudios se pueda profundizar en este análisis desde preguntas que orienten la reflexión de manera específica en la construcción del joven trabajador actual en distintos contextos latinoamericanos, donde están ocurriendo una serie de procesos de neoliberalización de las políticas del trabajo. 


\section{FUENTES DE CONSULTA}

Aguilera, Oscar, 2009, «Estudios sobre juventud en Chile: Coordenadas para un estado del Arte», Última Década, vol. 31, pp. 109-127.

Assusa, Gonzalo y María Gabriela Brandán, 2014, «'Salvar la generación perdida': gubernamentalidad, empleabilidad y cultura del trabajo. El caso de un programa de empleo para jóvenes en Argentina», Revista Sociología e Política, 22(49), pp. 157-174.

Atkinson, Paul y Amando Coffey, 2006, «Analysing documentary realities», en Silverman, David (ed.), Interpreting Qualitative Data, Londres, Sage, pp. 45-62.

Becker, Gary, 1993, Human capital: a theoretical and empirical analysis, with special reference to education, Londres, University of Chicago Press.

Brandán Zehnder, María Gabriela, 2014, «Juventud, trabajo y dispositivos estatales. Aportes críticos a la sociología de la juventud desde la perspectiva de la gubernamentalidad», Última década, 40(1), pp. 37-54.

Burchell, Graham, Colin Gordon y Peter Miller (eds.), 1991, The Foucault effect: Studies in Governmentality, Chicago, The University of Chicago Press.

Castro-Gómez, Santiago, 2010, Historia de la gubernamentalidad. Razón de Estado, liberalismo y neoliberalismo en Michel Foucault, Bogotá, Siglo del Hombre Editores.

Chertkovskaya, Ekaterina, Peter Watt, Stefan Tramer y Sverre Spoelstra, 2013, «Giving notice to employability», Ephemera, 13(4), pp. 701-716.

Congreso Nacional, 2009a, Mensaje de S. E la Presidenta de la República con que inicia un proyecto de ley que crea el subsidio al empleo. Historia de la Ley $N^{\circ} 20.338$. Crea el Subsidio al empleo.

2009b, Cámara de Diputados. Discusión en sala Subsidio al empleo juvenil. Primer trámite constitucional. Legislatura 357, Sesión 02. Fecha 12 de marzo, 2009. Historia de la Ley $N^{\circ}$ 20.338. Crea el Subsidio al empleo

2009c, Oficio de Ley a S. E. la Presidenta de la República, comunica texto aprobado por el Congreso Nacional. Fecha 19 de marzo, 2009. Historia de la Ley $N^{\circ}$ 20.338. Crea el Subsidio al empleo.

2009d, Firma proyecto de Ley Subsidio al empleo. Intervención de S. E. la Presidenta de la República Michelle Bachelet, en acto de firma del proyecto de Ley sobre Subsidio al empleo. Historia de la Ley No 20.338. Crea el Subsidio al empleo.

Consejo Asesor Presidencial Trabajo y Equidad, 2008, Hacia un Chile más justo: Trabajo, salario, competitividad y equidad social, Informe Final, CAPTE.

Deacon, Roger, 2007, «Pacifying the Planet: Norbert Elias on Globalization», Journal of Social and Political Theory, 113, pp. 76-96.

Dean, Mitchell, 1995, "Governing the unemployed self in an active society», Economy and Society, 24(4), pp. 559-583.

2007, «Governing societies», Londres, Open University Press/McGraw-Hill.

Diedrich, Andreas y Alexander Styhre, 2013, "Constructing the employable immigrant:

The uses of validation practices in Sweden», Ephemera, 13(4), pp. 759-783.

Du Gay, Paul, 1996, Consumption and identity at work, Londres, Sage.

Fairclough, Norman, 2010, Critical Discourse Analysis. The critical study of language, Londres, Longman. 
Fairclough, Norman y Ruth Wodak, 2000, «Análisis crítico del discurso», en Van Dijk, Teun (ed.), El discurso como interacción social, Guedisa, Barcelona, pp. 367-404.

Fejes, Andreas, 2010, «Dicourses on employability: Constituting the responsable citizen», Studies in continuing education, 32(2), pp. 89-102.

Flew, Terry, 2014, "Six theories of neoliberalism», Thesis Eleven, 122(1), pp. 49-71.

Foucault, Michel, 2009[1978], Seguridad, territorio y población, Argentina, Fondo de Cultura Económica. 2012a [1979], Nacimiento de la Biopolítica, Argentina, Fondo de Cultura Económica.

2012b, Tecnologías del Yo y otros textos a fines, España, Paidós.

Garsten, Christina y Kerstin Jacobsson, 2013, «Sorting people in and out: The plasticity of the categories of employability, work capacity and disability as technologies of government», Ephemera, 13(4), pp. 825-850.

Gobierno de Chile, 2014, Mensaje presidencial, 21 de mayo de 2014, <https://www.google.cl/webhp?sourceid=chrome-instant\&ion=1\&espv=2\&ie=UTF-8\#q=mensaje\%20 presidencial\%202014> [consulta: 18 de abril de 2016]

2015, Mensaje presidencial. 21 de mayo de 2015, <http://www.gob.cl/cuenta-publica/2015/2015_mensaje_presidencial.pdf>[consulta: 18 de abril de 2016]

Grinberg, Silvia, 2007, «Gubernamentalidad: estudios y perspectivas», Revista Argentina de Sociología, 5(8), pp. 95-110.

Holmqvist, Mikael, Christian Maravelias y Per Skalen, 2012, «ldentity regulation in neo-liberal societies: Constructing the "occupationally disabled" individual», Organization, 20(2), pp. 193-211.

Ibáñez, Schuda, 2005, El trabajo visto por los jóvenes chilenos: un análisis de las representaciones sociales de los jóvenes urbano populares, Montevideo, OIT/Cinterfor.

Jäger, Siegfried, 2003, «Discurso y conocimiento: Aspectos teóricos y metodológicos de la crítica del discurso y del análisis de dispositivos», en Ruth Wodak y Michael Meyer (comps.), Métodos de análisis crítico del discurso, Barcelona, Guedisa, pp. 61-100.

Kaasch, Alexandra, 2013, «Contesting contestation: Global social policy prescriptions on pension and health systems», Global Social Policy, 13(1), pp. 45-65.

Kotkas, Toomas, 2010, «Governing Health and Social Security in the Twenty-First Century: Active Citizenship Through the Right to Participate», Law Critique, vol. 21 pp. 163-182.

Miller, Peter y Nikolas Rose, 2009, Governing the present, Reino Unido, Polity Press.

Ministerio del Trabajo y previsión Social, 2009, «Ley N²0.338 Crea el subsidio al empleo», Publicación Diario Oficial. 1 de abril.

Mussetta, Paula, 2009, «Foucault y los anglofocultianos: una reseña del Estado y la gubernamentalidad», Revista Mexicana de Ciencias Políticas y Sociales, LI(205), pp. 37-55.

Organización Internacional del Trabajo, 2006, Tendencias mundiales de empleo juvenil, Ginebra, Oficina Internacional del Trabajo.

2010, Tendencias mundiales de empleo juvenil, edición especial sobre las repercusiones de la crisis económica mundial en los jóvenes, Ginebra, OIT-Oficina Internacional del Trabajo. 
Organización Internacional del Trabajo, 2013a, Tendencias mundiales de empleo juvenil 2013. Una generación en peligro, Ginebra, OIT.

2013b, Trabajo decente y juventud en América Latina 2013, Políticas para la acción, OIT-Oficina Regional para América Latina y el Caribe, Lima.

Parker, lan, 1996, «Discurso, cultura y poder en la vida cotidiana», en Ángel Gordo López y José Linaza (comps.), Psicologías, Discursos y Poder (PDP), Madrid, Visor, pp. 79-92.

Parker, Martin, George Cheney, Valery Fournier y Chris Land, 2014, The Routledge Companion to Alternative Organization, Londres, Routledge.

Pulido-Martínez, Hernán Camilo, 2008, «Produciendo trabajadores modernos. Conocimiento psicológico y el mundo del trabajo en el sur», Universitas Psychologica, 6(1), pp. 27-37.

2012, «El trabajador libre y la psicología del trabajo», en S. Trujillo-García y Hernán Camilo Pulido-Martínez (eds.), Libertad y psicología. Tensiones y debates desde Iberoamérica, Bogotá, Editorial Pontificia Universidad Javeriana, pp. 171-188

Rentería, Erico y Sigmar Malvezzi, 2008, «Empleabilidad, cambios y exigencias psicosociales en el trabajo», Universitas Psychologica, 7(2), pp. 9-24.

Rivera-Aguilera, Guillermo, 2015, «La construcción discursiva del joven trabajador: Un análisis crítico a los informes tendencias mundiales de empleo», Universitas Psychologica (artículo en revisión).

Rose, Nikolas, 1996, Inventig our selves. Psychology, power and personhood, Reino Unido, Cambridge University Press.

Rose, Nikolas, Pat O' Malley y Mariana Valverde, 2012, "Gubernamentalidad», Astrolabio, nueva época, 8, pp. 113-152.

Senellart, Michel, 2009, «Situación de los cursos», en Michel Foucault, Seguridad, territorio y población, Argentina, Fondo de Cultura Económica, pp. 417-453.

Servicio Nacional de Capacitación y Empleo (SENCE), 2015, Guías operativas 2015. Programa Más Capaz, Ministerio del Trabajo y Previsión, Gobierno de Chile, <http:// www.sence.cl/601/articles-3571_recurso_03.pdf> [consulta: 18 de abril de 2016]. 2014, Programa Más Capaz, Ministerio del Trabajo y Previsión, Gobierno de Chile, <http://www.sence.cl/portal/Perfil/Personas/Jovenes;Oportunidades/ Capacitacion/+Capaz/> [consulta:18 de abril de 2016].

Sisto, Vicente y Carla Fardella, 2011, «Nuevas políticas públicas, epocalismo e identidad: el caso de las políticas orientadas a los docentes en Chile», Revista de Estudios Universitarios, 37(1), pp. 123-141.

Standing, Guy, 2009, Work after globalization. Building Occupational Citizenship, Cheltenham, MPG Books Group.

Stecher, Antonio, 2010, «El análisis crítico del discurso como herramienta de investigación psicosocial del mundo del trabajo. Discusiones desde América Latina», Universitas Psychologica, 9(1), pp. 93-107.

2014, «Fairclough y el lenguaje en el Nuevo Capitalismo: Análisis de las dimensiones discursivas del mundo del trabajo», Psicoperspectivas, 13(3), pp. 19-19.

Stecher, Antonio y Lorena Godoy, 2014, Transformaciones del trabajo, subjetividad e identidades, Santiago de Chile, Ril Editores. 
Tadajewsky, Mark, Pauline Maclaran, E. Parson, Martin Parker, 2011, Key concepts in critical management studies, Londres, Sage.

Vargas-Monroy, Liliana y Margot Pujal, 2013, «Gubernamentalidad, dispositivos de género, raza y trabajo: la conducción de la conducta de las mujeres trabajadoras», Universitas Psychologica, 12(4), pp. 1255-1267.

Velasco, Andrésy Cristóbal Huneeus, 2011, Contra la desigualdad el empleo es la clave, Santiago, Mondadori.

Vesterberg, Victor, 2013, «Ethnicized un/employability: Problematized others and the shaping of advanced liberal subjets», Ephemera, 13(2), pp. 737-757.

Fecha de recepción: 2 de febrero de 2016

Fecha de aceptación: 19 de abril de 2016 